\title{
Diversity of Wild Pyrus communis Based on Microsatellite Analyses
}

\author{
Gayle M. Volk ${ }^{1}$, Christopher M. Richards, Adam D. Henk, and Ann A. Reilley \\ National Center for Genetic Resources Preservation, U.S. Department of Agriculture, 1111 S. Mason \\ Street, Fort Collins, CO 80521 \\ Nahla V. Bassil and Joseph D. Postman \\ National Clonal Germplasm Repository, U.S. Department of Agriculture, 33447 Peoria Road, Corvallis, \\ OR 97333
}

\begin{abstract}
AdDitional INDEX WORDS. pear, simple sequence repeat, genetic
Abstract. Edible european pears (Pyrus communis L. ssp. communis) are derived from wild relatives native to the Caucasus Mountain region and eastern Europe. Microsatellite markers (13 loci) were used to determine the relationships among 145 wild and cultivated individuals of $P$. communis maintained in the National Plant Germplasm System (NPGS). A Bayesian clustering method grouped the individual pear genotypes into 12 clusters. Pyrus communis ssp. caucasica (Fed.) Browicz, native to the Caucasus Mountains of Russia, Crimea, and Armenia, can be genetically differentiated from $P$. communis ssp. pyraster $\mathrm{L}$. native to eastern European countries. The domesticated pears cluster closely together and are most closely related to a group of genotypes that are intermediate to the $P$. communis ssp. pyraster and the $P$. communis ssp. caucasica groups. Based on the high number of unique alleles and heterozygosity in each of the 12 clusters, we conclude that genetic diversity of wild $P$. communis is not fully represented at the NPGS. Additional diversity may be present in seed accessions stored in the NPGS and more pear diversity could be captured through supplementary collection trips to eastern Europe, the Caucasus Mountains, and the surrounding countries.
\end{abstract}

Edible european pears were selected and bred from wild $P$. communis trees with small, nearly round, hard, gritty, sour, and astringent fruit (Hedrick, 1921). Large and medium-fruited edible pears were cultivated by Greeks and Romans as long as 2500 years ago and French monks and German botanists maintained ancient cultivars until the $16^{\text {th }}$ and $17^{\text {th }}$ centuries. Most modern cultivars originated from breeding efforts in Belgium and England in the 1700s (Hedrick, 1921; Lombard et al., 1980).

In the 1930s, Nicolai Vavilov recognized that Asia Minor (Trans-Caucasia, Iran, and Turkmenistan) represented a center of diversity for wild P. communis (Vavilov, 1994). The Caucasus Mountains provide diverse habitats that support highly variable germplasm (Vavilov, 1994). Over the past 50 years, seeds from wild $P$. communis trees were collected from natural or naturalized stands in the Caucasus Mountains, Crimea (Ukraine), Armenia, Turkey, the Balkans, and other European countries. While these individuals tend to have unacceptable fruiting qualities, they may provide valuable genetic diversity for the breeding of disease resistance to fire blight (Erwinia amylovora Burrill), pear psylla (Cacopsylla pyricola Foerster), and wooly pear aphid (Eriosoma pyricola Bak. and David.). Resistance to diseases and pests is a priority in pear breeding programs (Bell, 1982, 1992; van der Zwet et al., 1983; Westwood and Westigard, 1969). Multiple subspecies designations have been described for types of P. communis. Domesticated cultivars of $P$. communis ssp. communis have hybrid ancestry with wild $P$. communis subspecies and $P$. nivalis Jacquin, the snow pear. Also, $P$. communis ssp. pyraster and $P$. communis ssp. caucasica are thought to be most likely ancestors of the cultivated european pear (Challice and Westwood, 1973).

Received for publication 19 Sept. 2005. Accepted for publication 11 Feb. 2006 Mention of trade names or commercial products in this article is solely for the purpose of providing specific information and does not imply recommendation or endorsement by the U.S. Dept. of Agriculture.

'Corresponding author; e-mail gvolk@lamar.colostate.edu.
Phenotypically, $P$. communis ssp. pyraster and $P$. communis ssp. caucasica are similar (Aldasoro et al., 1996). These subspecies have been largely classified according to their geographical distribution. P. communis ssp. pyraster originates from areas west and south of the Black Sea such as the Balkan countries, Turkey and other European countries while wild forms of $P$. communis from areas around and east of the Caucasus Mountains (southwestern Russia, Crimea, Georgia, Armenia, and Azerbaijan) are classified as $P$. communis ssp. caucasica (Fig. 1). P. nivalis originates from Europe and Asia Minor. Selections and hybrids of P. nivalis species have been grown for hundreds of years throughout western Europe and especially in France and England for perry production (Challice and Westwood, 1973). Taxonomist Alfred Rehder considered P. korshinskyi Litv. (synonym P. bucharica Litv.) a related species of $P$. communis. . korshinskyi is native to central Asia and may have arisen from hybridization between $P$. communis and $P$. regelii Rehder (Rehder, 1940).

Taxonomic studies of species within the genus Pyrus L. show that differentiation is highly correlated with geographical origin. Using ordination analysis of morphological and chemotaxonomic characters, Challice and Westwood (1973) refined the concept of differentiation among the east Asian, west Asian, and European species and speculated on their phylogenetic origin. The authors were able to distinguish between the western European $P$. communis types, east Asian pea pears $[P$. betulifolia Bge., $P$. fauriei Schneider, $P$. dimorphophylla (Mak.) Koidz., P. calleryana Dcne.], and larger-fruited east Asian pears [P. pyrifolia (Burm. f.) Nakai, P. hondoensis Kikuchi \& Nakai, P. ussuriensis Maxim.]. The east Asian pears grouped with the wild European P. cordata (Desv.) Schneider, the wild north African P. longipes Henry, and the wild Asian P. pashia D. Don (Challice and Westwood, 1973). While work was able to distinguish broad taxonomic boundaries among Asian and European Pyrus species, attempts at delineating the subspecies of $P$. communis that are most closely related to domesticated pear were not successful. 
Microsatellites have been used for cultivar identification within both Asian ( $P$. pyrifolia) and European ( $P$. communis) pear species (Kimura et al., 2002; Yamamoto et al., 2002b, 2002c). In addition, many microsatellite loci show a high degree of synteny between apple (Malus Mill.) and Pyrus genomes (Yamamoto et al., 2001; Pierantoni et al., 2004), facilitating the use of apple markers in pear and simplifying the process of mapping the pear genome (Hayashi and Yamamoto, 2002; Hemmat et al., 2003; Katayama and Uematsu, 2003; Pierantoni et al., 2004; Yamamoto et al., 2001). Pear genetic linkage maps are valuable for breeding programs (Yamamoto et al., 2002a, 2005) as well as population genetic studies of diversity.

This study is aimed at examining the differentiation and diversity of $P$. communis ssp. pyraster and $P$. communis ssp. caucasica within an orchard collection at the National Plant Germplasm System(NPGS) National Clonal Germplasm Repository (NCGR) in Corvallis, Ore. The purpose of this work is to describe the extent of $P$. communis genetic diversity collected from Asia Minor and eastern Europe and use these data for germplasm management including suggestions for future collecting trips.

\section{Materials and Methods}

Plant materials. A total of $145 P$. communis individuals were used for this study. Exploration trips to eastern European and Caucasus regions have brought a diverse collection of wild P. communis clones and seeds to the United States. Much of this germplasm was channeled to the NCGR by H. Waterworth of the Plant Quarantine Station, Glenn Dale, Md., and M. Westwood, formerly a pomologist at Oregon State Univ. and is documented in the U.S. Dept. of Agriculture's Germplasm Resources Information Network (GRIN). Phenotypic and historical information for each individual is available by querying GRIN using plant introduction (PI) numbers listed in Table 1 (USDA, 2005).

Several historical exploration trips returned to the United States with wild Pyrus germplasm from the Trans-Caucasus region. In 1967, H. Brooks traveled to Crimea, Ukraine, and the Caucasus mountains between Teberda, Pyatigorsk, and Stavropol in the former USSR (Brooks, 1968). In 1977, D.R. Dewey and A.P. Plummer from Utah State Univ. collected wild P. communis seeds near Stavropol and Svetlograd, in the Russian Federation. Seedlots of $P$. communis were also collected from Armenia by S. Gasparian (Science Research Center of Viticulture, Fruit Growing, and Wine Making in Merdzavan, Armenia) in Fall 2003 and sent to the NCGR.

Collectors gathered seeds of diverse materials from eastern Europe. J.L. Creech and D.H. Scott collected P. communis seeds from trees in Moldova (formerly Moldavia, USSR) and Crimea, Ukraine (formerly USSR). T. van der Zwet published details of his collection trips to gather scions from named $P$. communis cultivar trees and landraces in Serbia, Yugoslavia, Romania, Macedonia, Czech Republic, and Poland (van der Zwet et al., 1983, 1989). T. Dimitrovski of the Univ. of Skopje, Macedonia, collected seeds from wild $P$. communis trees from Leva Reka, Stip, and Gorna Bosava-Kavadarci, Macedonia in 1969, 1971, and 1972, respectively, and provided cuttings to the NCGR in the 1980s. Additional P. communis seed collections were made in Turkey for M. Westwood by his colleague H. Olez in 1963 and in Kyrgyzstan by Maxine Thompson in 1994. Other individuals of $P$. communis selected for this project were unnamed largefruited types, rootstocks, and trees without specific collection localities, collected by M. Westwood and A. Rehder from the 1940s to 1960 s. Collection details for individuals are provided in Table 1 and Fig. 1.

Phenotypic observations. Many of the wild P. communis trees in the NPGS are more than 30 years old. Subspecies designations were assigned by the curator (J.D. Postman) using fruit and foliage characteristics as well as original geographic source data. Data were collected on fruit and leaves during the 2005 season at Corvallis, Ore. Quantitative phenotypic data included fruit size and peduncle length. Qualitative phenotypic data was collected for fruit shape, russetting, lenticel size, leaf shape, and peduncle thickness.

Molecular analysis. Duplicate samples of genomic DNA were isolated from young leaf tissue of 145 P. communis individuals using the PUREGENEkit (Gentra Systems, Minneapolis, Minn.). Thirteen microsatellite primers were selected from the literature (Table 2). These markers were unlinked and produced a maximum of two bands per reaction. Forward primers, labeled with either IRD 700 or IRD 800, were obtained from MWG-Biotech (High Point, N.C.). Unlabeled reverse primers were purchased from Integrated Technologies (Coralville, Iowa).

Polymerase chain reactions (PCR) were carried out in 15 $\mu \mathrm{L}$ total volume. For each reaction, 10 to $50 \mathrm{ng}$ DNA template and 0.3 to $0.7 \mathrm{pM}$ of primers were combined with 1.5 units Taq Polymerase (Promega, Madison,Wis.), 1X Promega magnesium free buffer [ $10 \mathrm{~mm}$ Tris- $\mathrm{HCl}, 50 \mathrm{~mm} \mathrm{KCl}$, and $0.1 \%$ Triton $\mathrm{X}-100$ (Sigma, St. Louis), $0.25 \mathrm{~mm} \mathrm{MgCl}_{2}$, and $0.25 \mathrm{~mm} \mathrm{dNTP} \mathrm{(Pro-}$ mega)]. PCR amplifications were carried out using a PTC200 thermocycler (MJ Research, Reno, Nev.) The PCR program had an initial denaturation step of $2 \mathrm{~min}$ at $95^{\circ} \mathrm{C}$ followed by 30 cycles of $30 \mathrm{~s}$ at $95^{\circ} \mathrm{C}, 30 \mathrm{~s}$ at the published primer-specific annealing temperature (Table 2 ), $15 \mathrm{~s}$ at $72{ }^{\circ} \mathrm{C}$ and ending with a final extension step of $2 \mathrm{~min}$ at $72{ }^{\circ} \mathrm{C}$. Completed $\mathrm{PCR}$ reactions were diluted 1:1 in 95\% formamide, 50 mm EDTA, bromophenol blue loading dye, and denatured at $95{ }^{\circ} \mathrm{C}$ for $3 \mathrm{~min}$. Gels $(6.5 \%$ LI-COR KB Plus acrylamide; LI-COR, Lincoln, Nebr.) were run in $1 \mathrm{X}$ TBE (89 mM Tris, $89 \mathrm{~mm}$ boric acid, $20 \mathrm{~mm}$ EDTA) buffer for $1 \mathrm{~h} 45 \mathrm{~min}$ at $1500 \mathrm{~V}, 40 \mathrm{~W}, 40 \mathrm{~mA}$, and $45^{\circ} \mathrm{C}$ on a LI-COR 4200 DNA Sequencer (LI-COR) Digital images were collected from the sequencer using LI-COR Saga Generation2 software and were manually analyzed using the Saga software. Alleles from replicate samples were examined at each locus, and when alleles for replicates were not identical, data for that locus were entered as "missing" in subsequent analyses. Allele sizes were calibrated by comparing values with data collected from P. pyrifolia (cultivar Hosui) and three Malus $\times$ domestica Borkh. individuals (PI 590184, PI 588853, PI 588850).

Data ANALYSES. We used complimentary approaches to cluster, estimate diversity and display genetic differentiation in the set of pear individuals using SSR data. Initially a Bayesian clustering analysis was conducted using the software STRUCTURE (Pritchard et al., 2000). This approach uses a model-based clustering algorithm to identify clusters of individuals that have distinctive allelic frequencies. Individuals are assigned to clusters based on their allelic frequencies without a priori information such as geographic origin or parentage. The model assumes $k$ groups, linkage equilibrium among markers, and Hardy-Weinberg equilibrium within a group. The parameter $k$ was determined by simulating a range of values of $k$ and the posterior probability of each value was assessed. Posterior probabilities were estimated using a Markov Chain, Monte Carlo (MCMC) method based on 50,000 iterations of each chain following a 30,000 iteration burnin period. Each MCMC chain for each value of $k$ (ranging from 1 
Table 1. Pyrus communis accessions maintained by the U.S. National Plant Germplasm System (NPGS) that were included in SSR analyses have been organized by assigned cluster (as determined using Bayesian clustering analyses). Plant introduction (PI) and Corvallis, Ore. Pyrus local (CPYR) identification numbers are provided. Pyrus communis ssp. communis assignments were based on fruit size and $P$. communis ssp. caucasica and P. communis ssp. pyraster assignments were based on collection location. Collection information includes donor (to the NPGS), collector, collection date, general source location, specific latitude and longitude, and approximate elevation. Q fit describes the membership coefficient of an individual for its assigned cluster.

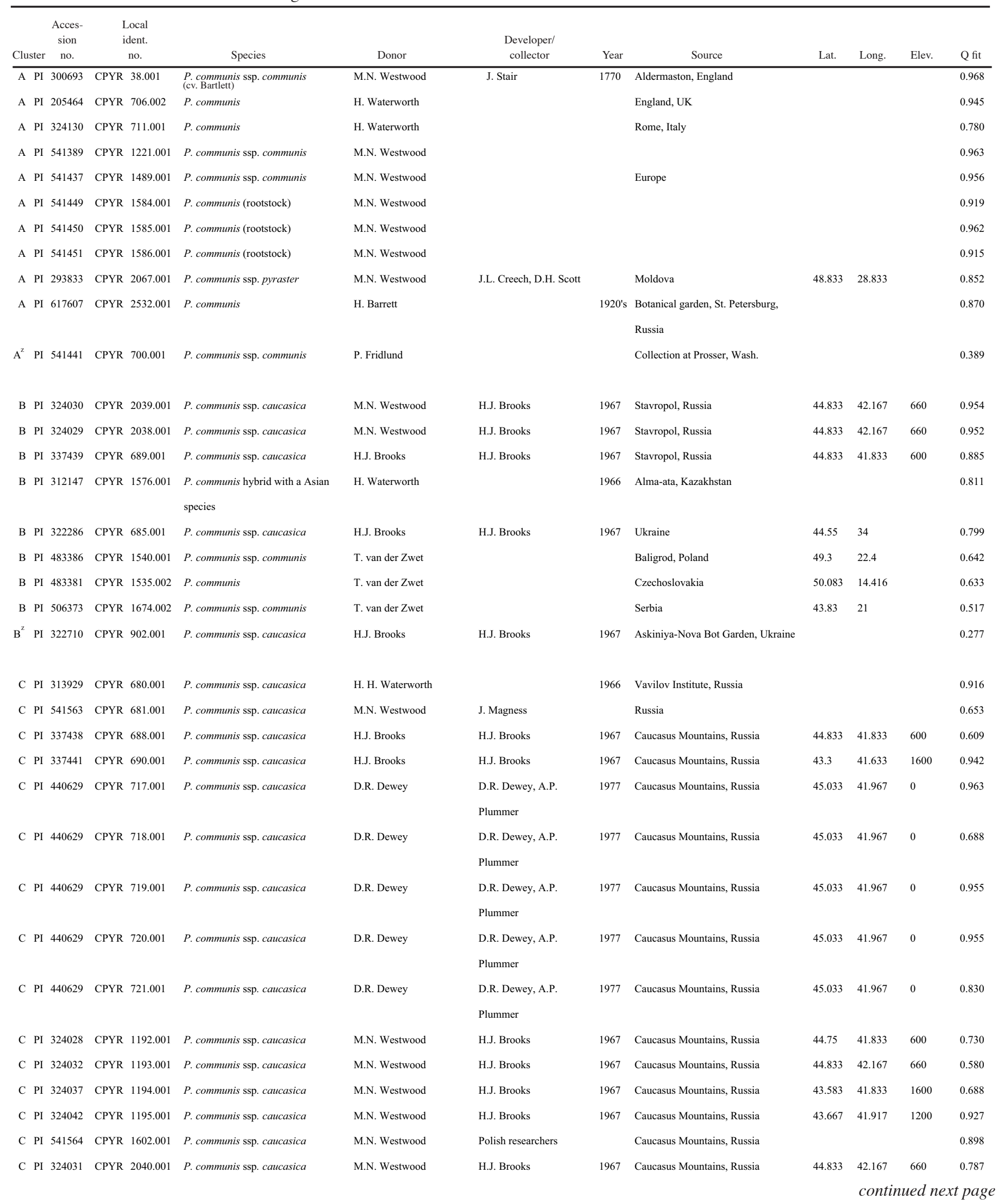


Table 1. Continued.

\begin{tabular}{|c|c|c|c|c|c|c|c|c|c|c|c|c|c|}
\hline Clus & ster & $\begin{array}{l}\text { Acces- } \\
\text { sion } \\
\text { no. }\end{array}$ & & $\begin{array}{l}\text { Local } \\
\text { dent. } \\
\text { no. }\end{array}$ & Species & Donor & $\begin{array}{l}\text { Developer/ } \\
\text { collector }\end{array}$ & Year & Source & Lat. & Long. & Elev. & Q fit \\
\hline $\mathrm{C}$ & PI & 324035 & CPYR & 2043.001 & P. communis ssp. caucasica & M.N. Westwood & H.J. Brooks & 1967 & Caucasus Mountains, Russia & 43.967 & 42.917 & 990 & 0.840 \\
\hline $\mathrm{C}$ & PI & 324038 & CPYR & 2045.001 & P. communis ssp. caucasica & M.N. Westwood & H.J. Brooks & 1967 & Caucasus Mountains, Russia & 43.583 & 41.833 & 1600 & 0.942 \\
\hline $\mathrm{C}$ & PI & 324043 & CPYR & 2049.001 & P. communis ssp. caucasica & M.N. Westwood & H.J. Brooks & 1967 & Caucasus Mountains, Russia & 43.667 & 41.917 & 1200 & 0.970 \\
\hline $\mathrm{C}$ & PI & 324044 & CPYR & 2050.001 & P. communis ssp. caucasica & M.N. Westwood & H.J. Brooks & 1967 & Caucasus Mountains, Russia & 43.667 & 41.917 & 1200 & 0.947 \\
\hline $\mathrm{C}$ & PI & 324047 & CPYR & 2053.001 & P. communis ssp. caucasica & M.N. Westwood & H.J. Brooks & 1967 & Caucasus Mountains, Russia & 44.167 & 42.5 & 540 & 0.851 \\
\hline $\mathrm{C}$ & PI & 324048 & CPYR & 2054.001 & P. communis ssp. caucasica & M.N. Westwood & H.J. Brooks & 1967 & Caucasus Mountains, Russia & 44.167 & 42.5 & 540 & 0.916 \\
\hline $\mathrm{C}$ & PI & 324050 & CPYR & 2056.001 & P. communis ssp. caucasica & M.N. Westwood & H.J. Brooks & 1967 & Caucasus Mountains, Russia & 44.133 & 43 & 930 & 0.540 \\
\hline $\mathrm{C}$ & PI & 293838 & CPYR & 2060.001 & P. communis ssp. caucasica & M.N. Westwood & J.L. Creech, D.H. Scott & & Crimea, Ukraine & 44.83 & 34.5 & & 0.642 \\
\hline $\mathrm{C}$ & PI & 617598 & CPYR & 2522.001 & P. communis ssp. korshinskyi & M. Thompson & M. Thompson & 1994 & $\begin{array}{l}\text { Experiment station, Ak-Terek, } \\
\text { Kyrgyzstan }\end{array}$ & 41.25 & 72.833 & 1600 & 0.885 \\
\hline $\mathrm{C}$ & PI & 617598 & CPYR & 2522.003 & P. communis ssp. korshinskyi & M. Thompson & M. Thompson & 1994 & $\begin{array}{l}\text { Experiment station, Ak-Terek, } \\
\text { Kyrgyzstan }\end{array}$ & 41.25 & 72.833 & 1600 & 0.951 \\
\hline $\mathrm{C}$ & PI & 617598 & CPYR & 2522.004 & P. communis ssp. korshinskyi & M. Thompson & M. Thompson & 1994 & $\begin{array}{l}\text { Experiment station, Ak-Terek, } \\
\text { Kyrgyzstan }\end{array}$ & 41.25 & 72.833 & 1600 & 0.714 \\
\hline $\mathrm{C}$ & PI & 617598 & CPYR & 2522.005 & P. communis ssp. korshinskyi & M. Thompson & M. Thompson & 1994 & $\begin{array}{l}\text { Experiment station, Ak-Terek, } \\
\text { Kyrgyzstan }\end{array}$ & 41.25 & 72.833 & 1600 & 0.971 \\
\hline $\mathrm{C}$ & PI & 617598 & CPYR & 2522.006 & P. communis ssp. korshinskyi & M. Thompson & M. Thompson & 1994 & $\begin{array}{l}\text { Experiment station, Ak-Terek, } \\
\text { Kyrgyzstan }\end{array}$ & 41.25 & 72.833 & 1600 & 0.918 \\
\hline $\mathrm{C}$ & PI & 617598 & CPYR & 2522.007 & P. communis ssp. korshinskyi & M. Thompson & M. Thompson & 1994 & $\begin{array}{l}\text { Experiment station, Ak-Terek, } \\
\text { Kyrgyzstan }\end{array}$ & 41.25 & 72.833 & 1600 & 0.891 \\
\hline $\mathrm{C}$ & PI & 617598 & CPYR & 2522.008 & P. communis ssp. korshinskyi & M. Thompson & M. Thompson & 1994 & $\begin{array}{l}\text { Experiment station, Ak-Terek, } \\
\text { Kyrgyzstan }\end{array}$ & 41.25 & 72.833 & 1600 & 0.882 \\
\hline $\mathrm{C}$ & PI & 617598 & CPYR & 2522.009 & P. communis ssp. korshinskyi & M. Thompson & M. Thompson & 1994 & $\begin{array}{l}\text { Experiment station, Ak-Terek, } \\
\text { Kyrgyzstan }\end{array}$ & 41.25 & 72.833 & 1600 & 0.798 \\
\hline $\mathrm{C}$ & PI & 638005 & CPYR & 2813.001 & P. communis ssp. caucasica & S. Gasparian & & 2003 & Armenia & 40.117 & 44.833 & & 0.725 \\
\hline $\mathrm{C}$ & PI & 638006 & CPYR & 2814.001 & P. communis ssp. caucasica & S. Gasparian & & 2003 & Armenia & 40.8425 & 44.3625 & & 0.611 \\
\hline $\mathrm{C}$ & PI & 638007 & CPYR & 2815.003 & P. communis ssp. caucasica & S. Gasparian & & 2003 & Armenia & 40.8425 & 44.3625 & & 0.834 \\
\hline$C^{2}$ & PI & 337437 & CPYR & 687.001 & P. communis ssp. caucasica & H.J. Brooks & H.J. Brooks & 1967 & Caucasus Mountains, Russia & 44.833 & 41.833 & 600 & 0.385 \\
\hline$C^{z}$ & PI & 324027 & CPYR & 2037.001 & P. communis ssp. caucasica & M.N. Westwood & H.J. Brooks & 1967 & Caucasus Mountains, Russia & 45.083 & 41.917 & 570 & 0.445 \\
\hline $\mathrm{C}^{\mathrm{z}}$ & PI & 293834 & CPYR & 2066.001 & P. communis ssp. caucasica & M.N. Westwood & J.L. Creech, D.H. Scott & & Crimea, Ukraine & & & & 0.470 \\
\hline$C^{2}$ & PI & 617598 & CPYR & 2522.002 & P. communis ssp. korshinskyi & M. Thompson & M. Thompson & 1994 & $\begin{array}{l}\text { Experiment station, Ak-Terek, } \\
\text { Kyrgyzstan }\end{array}$ & 41.25 & 72.833 & 1600 & 0.479 \\
\hline $\mathrm{D}$ & PI & 440630 & CPYR & 727.001 & P. communis ssp. caucasica & D.R. Dewey & $\begin{array}{l}\text { D.R. Dewey, A.P. } \\
\text { Plummer }\end{array}$ & 1977 & Caucasus Mountains, Russia & 44.683 & 42.483 & 400 & 0.867 \\
\hline $\mathrm{D}$ & PI & 440630 & CPYR & 728.001 & P. communis ssp. caucasica & D.R. Dewey & $\begin{array}{l}\text { D.R. Dewey, A.P. } \\
\text { Plummer }\end{array}$ & 1977 & Caucasus Mountains, Russia & 44.683 & 42.483 & 400 & 0.952 \\
\hline $\mathrm{D}$ & PI & 440630 & CPYR & 729.001 & P. communis ssp. caucasica & D.R. Dewey & $\begin{array}{l}\text { D.R. Dewey, A.P. } \\
\text { Plummer }\end{array}$ & 1977 & Caucasus Mountains, Russia & 44.683 & 42.483 & 400 & 0.970 \\
\hline $\mathrm{D}$ & PI & 541321 & CPYR & 731.001 & P. communis & D.R. Dewey & $\begin{array}{l}\text { D.R. Dewey, A.P. } \\
\text { Plummer }\end{array}$ & 1977 & Caucasus Mountains, Russia & 44.683 & 42.483 & 400 & 0.971 \\
\hline E & PI & 440631 & CPYR & 694.001 & P. communis ssp. caucasica & D.R. Dewey & $\begin{array}{l}\text { D.R. Dewey, A.P. } \\
\text { Plummer }\end{array}$ & 1977 & Svetlograd, Russia & 45.067 & 43.033 & 300 & 0.955 \\
\hline E & PI & 440631 & CPYR & 695.001 & P. communis ssp. caucasica & D.R. Dewey & $\begin{array}{l}\text { D.R. Dewey, A.P. } \\
\text { Plummer }\end{array}$ & 1977 & Svetlograd, Russia & 45.067 & 43.033 & 300 & 0.965 \\
\hline $\mathrm{E}$ & PI & 440632 & CPYR & 697.001 & P. communis ssp. caucasica & D.R. Dewey & $\begin{array}{l}\text { D.R. Dewey, A.P. } \\
\text { Plummer }\end{array}$ & 1977 & Svetlograd, Russia & 45.067 & 43.033 & 300 & 0.906 \\
\hline
\end{tabular}

continued next page 
Table 1. Continued.

\begin{tabular}{|c|c|c|c|c|c|c|c|c|c|c|c|c|c|}
\hline Clus & ster & $\begin{array}{c}\text { Acces- } \\
\text { sion } \\
\text { no. }\end{array}$ & & $\begin{array}{l}\text { Local } \\
\text { ident. } \\
\text { no. }\end{array}$ & Species & Donor & $\begin{array}{l}\text { Developer/ } \\
\text { collector }\end{array}$ & Year & Source & Lat. & Long. & Elev. & Q fit \\
\hline $\mathrm{E}$ & PI & 440632 & CPYR & 697.001 & P. communis ssp. caucasica & D.R. Dewey & D.R. Dewey, A.P. & 1977 & Svetlograd, Russia & 45.067 & 43.033 & 300 & 0.906 \\
\hline \multirow{3}{*}{\multicolumn{2}{|c|}{ E PI }} & \multirow{3}{*}{440632} & \multirow{3}{*}{ CPYR } & \multirow{3}{*}{698.001} & \multirow{3}{*}{ P. communis ssp. caucasica } & \multirow{3}{*}{ D.R. Dewey } & Plummer & \multirow{3}{*}{1977} & \multirow{3}{*}{ Svetlograd, Russia } & \multirow{3}{*}{45.067} & \multirow{3}{*}{43.033} & \multirow{3}{*}{300} & \multirow{3}{*}{0.961} \\
\hline & & & & & & & D.R. Dewey, A.P. & & & & & & \\
\hline & & & & & & & Plummer & & & & & & \\
\hline$E^{2}$ & PI & 293842 & CPYR & 2059.001 & P. communis ssp. pyraster & M.N. Westwood & J.L. Creech, D.H. Scott & & Moldova & 48.833 & 28.833 & 300 & 0.374 \\
\hline $\mathrm{F}$ & PI & 638004 & CPYR & 2812.002 & P. communis ssp. caucasica & S. Gasparian & & 2003 & Armenia & 40.8425 & 44.3625 & & 0.731 \\
\hline $\mathrm{F}$ & PI & 638005 & CPYR & 2813.002 & P. communis ssp. caucasica & S. Gasparian & & 2003 & Armenia & 40.8425 & 44.3625 & & 0.945 \\
\hline $\mathrm{F}$ & PI & 638005 & CPYR & 2813.003 & P. communis ssp. caucasica & S. Gasparian & & 2003 & Armenia & 40.8425 & 44.3625 & & 0.536 \\
\hline $\mathrm{F}$ & PI & 638005 & CPYR & 2813.004 & P. communis ssp. caucasica & S. Gasparian & & 2003 & Armenia & 40.8425 & 44.3625 & & 0.944 \\
\hline $\mathrm{F}$ & PI & 638007 & CPYR & 2815.001 & P. communis ssp. caucasica & S. Gasparian & & 2003 & Armenia & 40.8425 & 44.3625 & & 0.974 \\
\hline $\mathrm{F}$ & PI & 638007 & CPYR & 2815.002 & P. communis ssp. caucasica & S. Gasparian & & 2003 & Armenia & 40.8425 & 44.3625 & & 0.727 \\
\hline $\mathrm{F}$ & PI & 638008 & CPYR & 2816.001 & P. communis ssp. caucasica & S. Gasparian & & 2003 & Armenia & 40.8425 & 44.3625 & & 0.958 \\
\hline $\mathrm{F}$ & PI & 638008 & CPYR & 2816.002 & P. communis ssp. caucasica & S. Gasparian & & 2003 & Armenia & 40.8425 & 44.3625 & & 0.947 \\
\hline $\mathrm{F}$ & PI & 638008 & CPYR & 2816.003 & P. communis ssp. caucasica & S. Gasparian & & 2003 & Armenia & 40.8425 & 44.3625 & & 0.980 \\
\hline $\mathrm{F}$ & PI & 638008 & CPYR & 2816.004 & P. communis ssp. caucasica & S. Gasparian & & 2003 & Armenia & 40.8425 & 44.3625 & & 0.978 \\
\hline $\mathrm{F}$ & PI & 638008 & CPYR & 2816.005 & P. communis ssp. caucasica & S. Gasparian & & 2003 & Armenia & 40.8425 & 44.3625 & & 0.832 \\
\hline $\mathrm{F}$ & PI & 638008 & CPYR & 2816.006 & P. communis ssp. caucasica & S. Gasparian & & 2003 & Armenia & 40.8425 & 44.3625 & & 0.955 \\
\hline $\mathrm{F}$ & PI & 638008 & CPYR & 2816.007 & P. communis ssp. caucasica & S. Gasparian & & 2003 & Armenia & 40.8425 & 44.3625 & & 0.974 \\
\hline $\mathrm{F}$ & PI & 638008 & CPYR & 2816.008 & P. communis ssp. caucasica & S. Gasparian & & 2003 & Armenia & 40.8425 & 44.3625 & & 0.976 \\
\hline $\mathrm{F}$ & PI & 638008 & CPYR & 2816.009 & P. communis ssp. caucasica & S. Gasparian & & 2003 & Armenia & 40.8425 & 44.3625 & & 0.976 \\
\hline $\mathrm{F}$ & PI & 638008 & CPYR & 2816.010 & P. communis ssp. caucasica & S. Gasparian & & 2003 & Armenia & 40.8425 & 44.3625 & & 0.980 \\
\hline G & PI & 541291 & CPYR & 693.001 & P. communis ssp. pyraster & M.N. Westwood & H. Olez & 1963 & Turkey & 38.2 & 28.6 & & 0.928 \\
\hline G & PI & 541292 & CPYR & 710.001 & P. communis ssp. pyraster & M.N. Westwood & H. Olez & 1963 & Turkey & & & & 0.907 \\
\hline G & PI & 541323 & CPYR & 712.001 & P. communis ssp. pyraster & M.N. Westwood & H. Olez & 1963 & Turkey & & & & 0.915 \\
\hline G & PI & 369881 & CPYR & 986.001 & P. communis ssp. pyraster & M.N. Westwood & T. Dimitrovski & 1971 & Macedonia & 41.733 & 22.183 & & 0.729 \\
\hline G & PI & 369881 & CPYR & 986.002 & P. communis ssp. pyraster & M.N. Westwood & T. Dimitrovski & 1971 & Macedonia & 41.733 & 22.183 & & 0.522 \\
\hline G & PI & 369881 & CPYR & 986.005 & P. communis ssp. pyraster & M.N. Westwood & T. Dimitrovski & 1971 & Macedonia & 41.733 & 22.183 & & 0.708 \\
\hline G & PI & 541391 & CPYR & 1248.001 & P. communis ssp. pyraster & M.N. Westwood & H. Olez & 1963 & Turkey & 38.2 & 28.6 & & 0.937 \\
\hline G & PI & 541392 & CPYR & 1249.001 & P. communis ssp. pyraster & M.N. Westwood & H. Olez & 1963 & Turkey & 38.2 & 28.6 & & 0.927 \\
\hline G & PI & 541393 & CPYR & 1250.001 & P. communis ssp. pyraster & M.N. Westwood & H. Olez & 1963 & Turkey & 38.2 & 28.6 & & 0.578 \\
\hline G & PI & 541394 & CPYR & 1251.002 & P. communis ssp. pyraster & M.N. Westwood & & & France & & & & 0.684 \\
\hline G & PI & 541395 & CPYR & 1252.001 & P. communis ssp. pyraster & M.N. Westwood & H. Olez & 1963 & Turkey & 38.2 & 28.6 & & 0.875 \\
\hline G & PI & 541435 & CPYR & 1465.001 & P. communis ssp. pyraster & M.N. Westwood & H. Olez & 1963 & Turkey & 38.2 & 28.6 & & 0.936 \\
\hline G & PI & 541436 & CPYR & 1466.001 & P. communis ssp. pyraster & M.N. Westwood & H. Olez & 1963 & Turkey & 38.2 & 28.6 & & 0.841 \\
\hline G & PI & 617521 & CPYR & 1467.001 & P. communis ssp. pyraster & M.N. Westwood & H. Olez & 1963 & Turkey & 38.2 & 28.6 & & 0.681 \\
\hline G & PI & 506380 & CPYR & 1671.001 & P. communis ssp. pyraster & T. van der Zwet & & & Valsoara, Romania & & & & 0.585 \\
\hline G & PI & 541490 & CPYR & 2057.001 & P. communis ssp. pyraster & M.N. Westwood & H. Olez & 1963 & Turkey & & & & 0.868 \\
\hline $\mathrm{H}$ & PI & 349026 & CPYR & 989.001 & P. communis ssp. pyraster & M.N. Westwood & T. Dimitrovski & 1969 & Izvor, Macedonia & 41.555 & 41.833 & & 0.923 \\
\hline $\mathrm{H}$ & PI & 349026 & CPYR & 989.002 & P. communis ssp. pyraster & M.N. Westwood & T. Dimitrovski & 1969 & Izvor, Macedonia & 41.555 & 41.833 & & 0.957 \\
\hline $\mathrm{H}$ & PI & 349026 & CPYR & 989.003 & P. communis ssp. pyraster & M.N. Westwood & T. Dimitrovski & 1969 & Izvor, Macedonia & 41.555 & 41.833 & & 0.831 \\
\hline $\mathrm{H}$ & PI & 349026 & CPYR & 989.004 & P. communis ssp. pyraster & M.N. Westwood & T. Dimitrovski & 1969 & Izvor, Macedonia & 41.555 & 41.833 & & 0.901 \\
\hline $\mathrm{H}$ & PI & 349026 & CPYR & 989.005 & P. communis ssp. pyraster & M.N. Westwood & T. Dimitrovski & 1969 & Izvor, Macedonia & 41.555 & 41.833 & & 0.971 \\
\hline $\mathrm{H}$ & PI & 349026 & CPYR & 989.006 & P. communis ssp. pyraster & M.N. Westwood & T. Dimitrovski & 1969 & Izvor, Macedonia & 41.555 & 41.833 & & 0.597 \\
\hline $\mathrm{H}$ & PI & 349026 & CPYR & 989.007 & P. communis ssp. pyraster & M.N. Westwood & T. Dimitrovski & 1969 & Izvor, Macedonia & 41.555 & 41.833 & & 0.557 \\
\hline
\end{tabular}


Table 1. Continued.

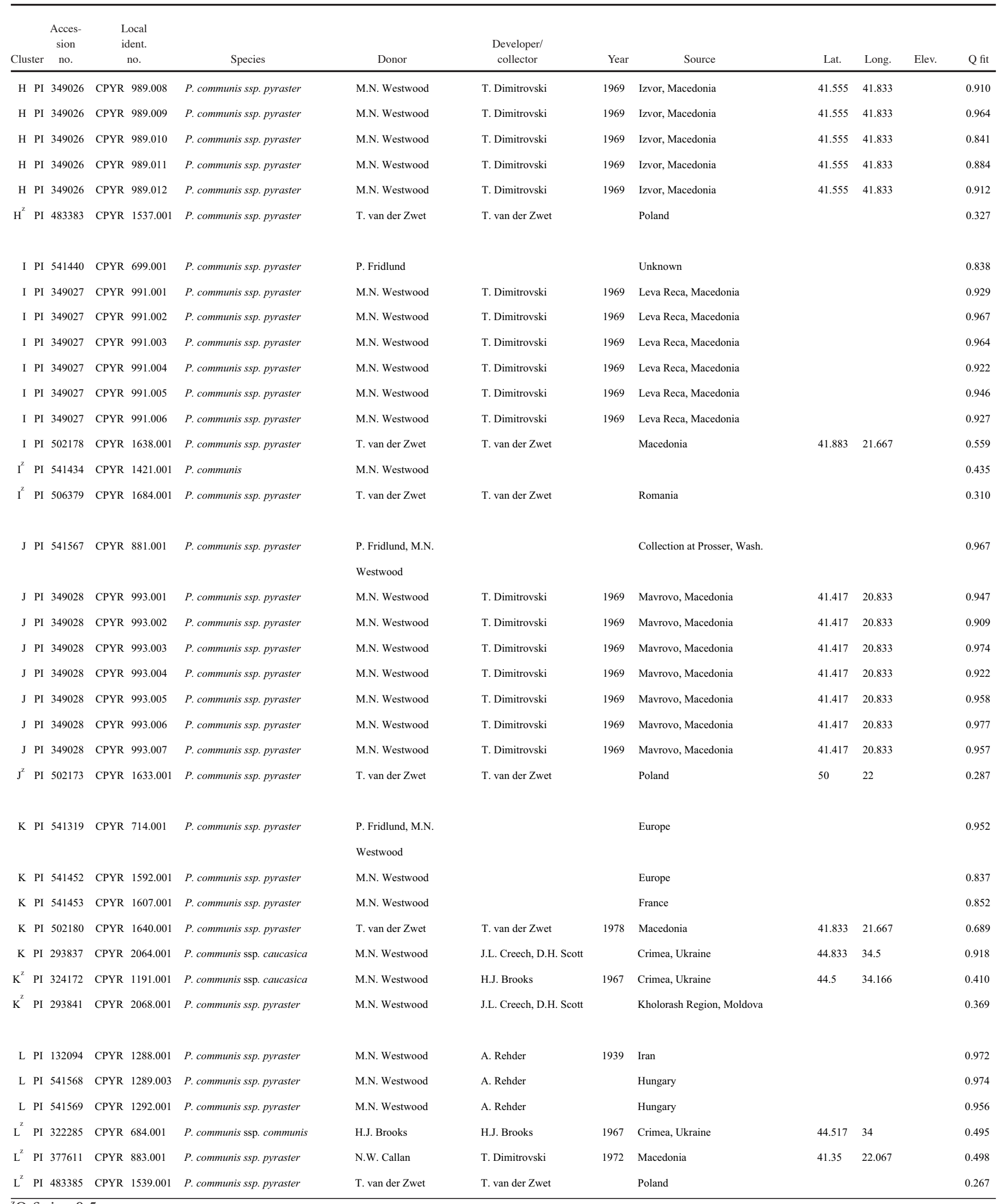

${ }^{\mathrm{Z}} \mathrm{Q}$ fit is $<0.5$. 
Table 2. Microsatellite loci were used to amplify pear DNA. The original cited name, range of allele sizes (in base pairs), number of amplified alleles, genetic mapping information, and original citations are provided for each locus.

\begin{tabular}{lcccc}
\hline Microsatellite & $\begin{array}{c}\text { Allele size } \\
(\mathrm{bp})\end{array}$ & $\begin{array}{c}\text { Alleles } \\
\text { amplified (no.) }\end{array}$ & $\begin{array}{c}\text { Chromosomal } \\
\text { linkage group no. }\end{array}$ & Source \\
\hline NH009b & $134-154$ & 9 & 13 & Yamamoto et al., 2005 \\
NH015a & $95-127$ & 14 & 17 & Yamamoto et al., 2005 \\
CH01D08 & $226-300$ & 14 & 15 & Liebhard et al., 2002, 2003 \\
CH01D09 & $118-172$ & 23 & 12 & Liebhard et al., 2002, 2003 \\
CH01F07A & $176-226$ & 19 & 10 & Liebhard et al., 2002, 2003 \\
CH01H01 & $97-123$ & 11 & 17 & Liebhard et al., 2002, 2003 \\
CH02B10 & $113-159$ & 18 & 2 & Liebhard et al., 2002, 2003 \\
CH02D12 & $213-255$ & 23 & 11 & Liebhard et al., 2002, 2003 \\
CH05E03 & $151-221$ & 18 & 2 & Liebhard et al., 2002, 2003 \\
GD12 & $136-170$ & 26 & 10 & Hemmat et al., 2003 \\
GD142 & $136-188$ & 18 & 3 & Hemmat et al., 2003 \\
GD96 & $141-175$ & 9 & 1 & Hemmat et al., 2003 \\
GD147 & $120-148$ & 4 & Hemmat et al., 2003 \\
\hline
\end{tabular}

Table 3. Descriptive information is provided for each of the 12 clusters of genotypes identified by Bayesian clustering analyses. Summary statistics are given for each cluster as a whole. Summary statistics include the number of individuals in each cluster (N), Nei's gene diversity, the number of alleles unique to the identified cluster and the total number of alleles scored across all microsatellite loci. Allelic richness is a scaled measure of allelic diversity that controls for cluster size.

\begin{tabular}{|c|c|c|c|c|c|c|c|c|c|c|c|c|c|c|c|c|c|c|c|}
\hline \multirow[b]{2}{*}{ Cluster } & \multirow[b]{2}{*}{ Origin } & \multirow[b]{2}{*}{$\mathrm{N}$} & \multirow{2}{*}{$\begin{array}{c}\text { Gene } \\
\text { diversity }\end{array}$} & \multirow{2}{*}{$\begin{array}{l}\text { Unique } \\
\text { alleles } \\
\text { (no.) }\end{array}$} & \multicolumn{13}{|c|}{ Alleles at each SSR locus (no.) } & \multirow{2}{*}{$\begin{array}{c}\text { Total } \\
\text { alleles } \\
\text { (no.) }\end{array}$} & \multirow[b]{2}{*}{$\begin{array}{c}\text { Allelic } \\
\text { richness }\end{array}$} \\
\hline & & & & & CH01D08 & CH01d09 & CH01F07A & Ch01h01 & $\mathrm{CH} 02 \mathrm{~b} 10$ & $\mathrm{CH} 02 \mathrm{~d} 12$ & $\mathrm{CH} 05 \mathrm{e} 03$ & GD12 & GD96 & GD142 & GD147 & NH009b & $\overline{\mathrm{NH} 015 \mathrm{a}}$ & & \\
\hline $\mathrm{A}$ & Domesticated & 11 & 0.64 & 3 & 7 & 6 & 6 & 4 & 6 & 6 & 8 & 5 & 5 & 6 & 2 & 2 & 7 & 70 & 3.28 \\
\hline B & Various & 9 & 0.75 & 5 & 5 & 7 & 8 & 7 & 8 & 8 & 8 & 7 & 8 & 8 & 5 & 6 & 7 & 92 & 3.99 \\
\hline $\mathrm{C}$ & $\begin{array}{l}\text { Caucasus, } \\
\text { Ukraine }\end{array}$ & 38 & 0.68 & 11 & 7 & 16 & 10 & 7 & 8 & 17 & 22 & 11 & 13 & 15 & 7 & 4 & 8 & 145 & 2.90 \\
\hline $\mathrm{D}$ & Caucasus & 4 & 0.49 & 0 & 1 & 4 & 3 & 2 & 2 & 4 & 2 & 3 & 3 & 4 & 4 & 1 & 3 & 36 & 3.64 \\
\hline E & Caucasus & 5 & 0.60 & 0 & 2 & 6 & 3 & 2 & 4 & 8 & 2 & 2 & 4 & 4 & 4 & 2 & 4 & 47 & 3.52 \\
\hline $\mathrm{F}$ & Armenia & 17 & 0.57 & 3 & 3 & 6 & 5 & 6 & 5 & 9 & 7 & 4 & 5 & 4 & 2 & 3 & 4 & 63 & 2.52 \\
\hline G & $\begin{array}{l}\text { Turkey, } \\
\text { Macedonia }\end{array}$ & 16 & 0.75 & 8 & 8 & 11 & 8 & 6 & 5 & 10 & 11 & 4 & 11 & 16 & 5 & 6 & 6 & 107 & 3.93 \\
\hline $\mathrm{H}$ & Macedonia & 13 & 0.67 & 7 & 3 & 8 & 7 & 6 & 7 & 11 & 9 & 4 & 5 & 9 & 7 & 5 & 6 & 87 & 3.47 \\
\hline I & Macedonia & 10 & 0.70 & 7 & 5 & 8 & 6 & 5 & 8 & 7 & 5 & 4 & 6 & 8 & 4 & 5 & 6 & 77 & 3.35 \\
\hline $\mathrm{J}$ & Macedonia & 9 & 0.62 & 0 & 3 & 6 & 5 & 3 & 6 & 8 & 5 & 1 & 2 & 4 & 5 & 4 & 5 & 57 & 3.93 \\
\hline $\mathrm{K}$ & $\begin{array}{l}\text { Europe, } \\
\text { various }\end{array}$ & 7 & 0.74 & 3 & 5 & 8 & 5 & 4 & 8 & 9 & 9 & 4 & 7 & 4 & 5 & 3 & 8 & 79 & 3.14 \\
\hline $\mathrm{L}$ & Various & 6 & 0.62 & 3 & 4 & 8 & 5 & 5 & 4 & 3 & 5 & 2 & 3 & 6 & 4 & 5 & 7 & 61 & 2.82 \\
\hline
\end{tabular}

to 40) was run 10 times. The method allows for individuals with ancestry from more than one group. These individuals are fractionally assigned to multiple groups using a membership coefficient (Q) which sums to 1 across all groups. Individual assignments can vary across runs when there is a weak genetic basis for assigning an individual to a cluster. To address this variation, we ran 100 separate MCMC chains at the most probable value of $k$ to look for similarity among assignments (Rosenberg et al., 2002).

Descriptive statistics, including variation between groups $\left(\mathrm{F}_{\mathrm{st}}\right)$, and diversity within groups including Nei's gene diversity (Nei, 1987), number of polymorphic alleles and allelic richness (El Mousadik and Petit, 1996) were estimated from genotypic data using the software package GDA (Lewis and Zaykin, 2001) and FSTAT (Goudet, 1995). Pairwise $\mathrm{F}_{\mathrm{st}}$ values were tested for significance using a permutation test. Analysis of molecular variance (AMOVA) was carried out using the software ARLEQUIN ver. 2.0 (Schneider et al., 2000).

Groups were plotted as nodes in a minimum spanning network using MINSPNET, a module within ARLEQUIN (Schneider et al., 2000) using pairwise $\mathrm{F}_{\mathrm{st}}$ values as the distance metric. The network display has a number of advantages over a bifurcation tree structure, especially when the pedigrees of the individuals are reticulate (characterized by ancestral interspecific hybridization). The minimum spanning tree was manually drawn using computer outputs.

\section{Results}

Genotypic data were collected for 145 P. communis individuals. Microsatellites provided between 9 and 33 alleles per locus (Table 2). A total of 235 microsatellite alleles were scored within the dataset.

Phenotypic ObServations. Morphological data were used in an attempt to differentiate individuals according to $P$. communis ssp. communis (domesticated), P. communis ssp. pyraster (south and west of the Black Sea), and $P$. communis ssp. caucasica (north and east of the Black Sea) (Fig. 1). Floral and fruit characteristics could not distinguish between $P$. communis ssp. pyraster and $P$. 
communis ssp. caucasica [data not shown, but available online (USDA, ARS, National Genetic Resources Program, 2005)]. The $P$. communis ssp. communis individuals (including hybrids between cultivated and wildtypes) have larger fruit than individuals in the other subspecies of $P$. communis. In 2005, accessions PI 300693, PI 541389, PI 541437, PI 293833, PI 324030, PI 324029, PI 337439, PI 322286, and PI 506373 had large fruit, characteristic of cultivated-type pears.

Genetic ANALYSIS. Posterior probabilities of Bayesian clustering analysis across a range of $k$ identified $(k=12)$ as the most probable clusters within the dataset. Among the subsequent 100 separate MCMC chains run with $k=12$, individual assignments to groups were highly correlated $(>0.85)$ among runs. All clusters showed varying degrees of admixture reflecting the relatedness of individuals (such as sibling collections) (Table 3). Sixteen individuals ( $11 \%$ ) had membership coefficients, $Q$, less than 0.5 . While the affinity of these individuals to their assigned cluster was low, their placement in a cluster reflects consistent assignments among runs and represents the best fit of the data. Phenotypic observations of fruit and leaf characteristics of these individuals revealed that seven of these 16 individuals exhibited large fruit or serrated leaf margins. Hybridization between wildtype $P$. communis and domesticated $P$. communis ssp. communis could result in large fruit, while hybridization between $P$. communis and another Pyrus species (such as P. nivalis) could result in serrated leaf margins and low membership coefficients.

Genetic diversity data were calculated by cluster. Nei's gene diversity ranged from 0.49 to 0.74 , revealing a high level of heterozygous individuals. Each of the 12 clusters identified was significantly differentiated with an average $F_{\text {st }}$ value of 0.18 . AMOVA results indicated that the within cluster variance component accounted for $82 \%$ of total variation, indicative of a highly variable, outcrossing species (Table 3).
The number of alleles represented across each of the 13 molecular markers was high (235), with most markers providing many diverse alleles. We used allelic diversity (El Mousadik and Petit, 1996) which employs a statistical scaling method to make direct comparisons among clusters composed of different numbers of individuals $(N)$. In this data set, the sample size is set to the smallest cluster size where $N=4$. Cluster B showed the highest allelic diversity, followed by clusters $\mathrm{G}$ and J. Cluster size did not correlate to measures of genetic diversity. The largest cluster (cluster $\mathrm{C}$ with $N=38$ individuals) had one of the lowest measures of allelic richness based upon Nei's gene diversity score (Table 3).

Clustering of individuals. Outputs were used to create a minimum spanning tree that graphically displays the genetic differentiation among the 12 clusters identified in Bayesian analysis (Fig. 2). Each node in the network corresponds to a particular cluster of genotypes. Distances between each connected cluster on the diagram represent $F_{s t}$ values. Each node is displayed as a pie chart where node diameters correlate to the number of individuals within the cluster(Fig. 2). Pie chart shading represents the number of individuals identified as either $P$. communis ssp. communis, $P$. communis ssp. pyraster, or $P$. communis ssp. caucasica. Pairwise $\mathrm{F}_{\text {st }}$ values among clusters were significant at $\alpha=0.05$.

The broad structure of the network shows a central cluster (B) connected to genotypes of domesticated lineages (node A) and two other clusters. Each of these clusters ( $G$ and $C$ ) forms the center of two distinct star groups. Clusters $\mathrm{C}-\mathrm{F}$ (predominantly $P$. communis ssp. caucasica) are genetically differentiated from clusters G-L (predominantly $P$. communis ssp. pyraster). These data support the genetic differentiation of $P$. communis ssp. pyraster from $P$. communis ssp. caucasica.

Clusters A and B both include individuals with large domesticated-type fruit characteristic of $P$. communis ssp. communis. Cluster A contains mostly domesticated cultivars including the

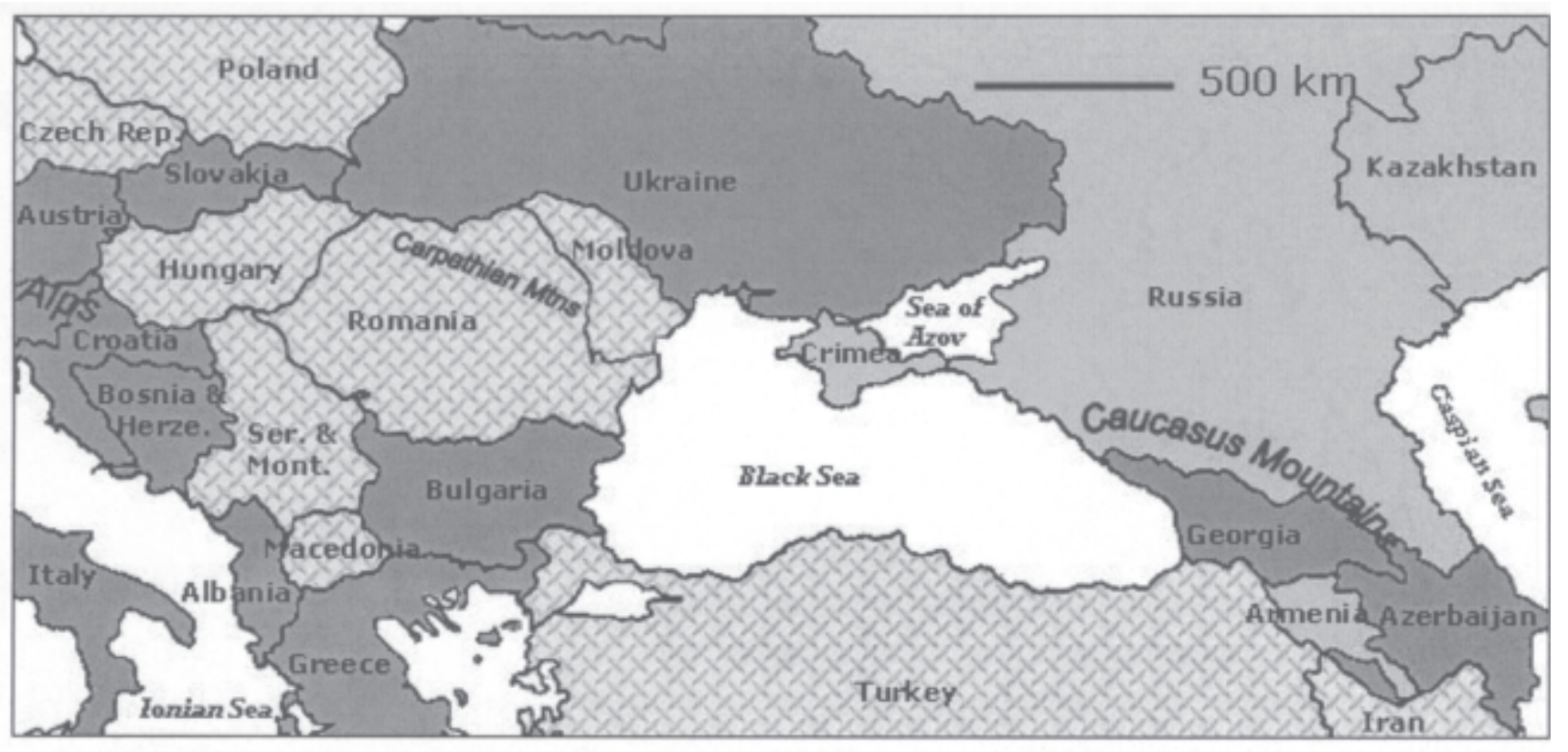

$$
\begin{aligned}
& \square \text { Pyns communis ssp. pyraster } \\
& \square \text { Pyrus communis ssp. caucasica } \\
& \square \text { Not sampled }
\end{aligned}
$$

Fig. 1. Map of the countries of origin for the wild Pyrus communis individuals from the U.S. National Plant Germplasm System collection. 


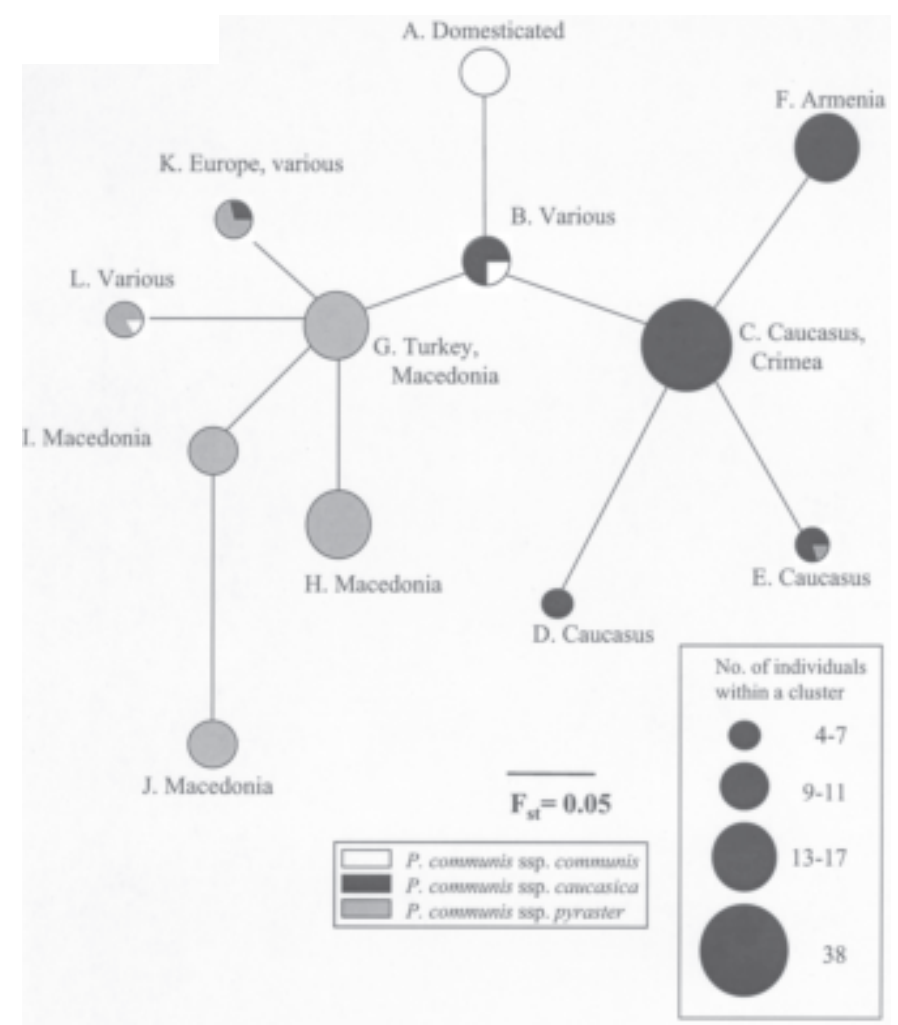

Fig. 2. A minimum spanning network for the wild Pyrus communis individuals from the U.S. National Plant Germplasm System collection. Distances among connected nodes represent $\mathrm{F}_{\mathrm{st}}$ values. Pie chart nodes represent proportions of individuals from $P$. communis ssp. communis, $P$. communis ssp. caucasica, and $P$. communis ssp. pyraster in the clusters identified through Bayesian analysis. The diameter of each node reflects the number of individuals within the cluster.

cultivar Bartlett as well as some domesticated rootstock cultivars. Cluster B contains a mixture of $P$. communis ssp. communis and $P$. communis ssp. caucasica. Individuals in cluster B were collected by Brooks from the northern side of the Caucasus Mountains and Ukraine and by van der Zwet in Poland, Serbia, and the Czech Republic (Table 1).

Clusters $\mathrm{C}-\mathrm{F}$ contain individuals classified as $P$. communis ssp. caucasica that were acquired from several collection trips in the Armenia, Crimea, and Caucasus Mountain regions. These include collection trips by Creech and Scott in Crimea, Brooks as well as Dewey and Plummer in the Caucasus Mountains of Russia and Thompson in Kyrgyzstan (Table 1). Thompson's seedlot from Kyrgyzstan was originally classified as $P$. communis ssp. korshinskyi; however, genotypic data suggest that these individuals should be reclassified as $P$. communis ssp. caucasica.

Clusters G-L contain accessions mostly classified as P. communis ssp. pyraster. These clusters include individuals from European, Balkan, and western Turkish locations. Cluster G contains 10 individuals from Turkey, a sibship of three individuals from Macedonia, and seedling selections from France and Romania. Clusters $\mathrm{H}$, I, and $\mathrm{J}$ are primarily comprised of sibling populations from Macedonia. Cluster Hhas 12 siblings from Izvor, Macedonia, and one individual from Poland. Cluster I includes six siblings from Leva Reca, Macedonia, one individual each from van der Zwet's trips to Macedonia and Romania, and two individuals of unknown origins. Cluster J contains seven siblings from Mavrovo, Macedonia, and two other individuals of unknown origin. Cluster $\mathrm{K}$ is comprised of a cultivar of seedling selections donated by
Westwood that came from seedlots from France, Europe, Crimea, Macedonia, and Moldova. Cluster L originates from a variety of sources. Three individuals came from A. Rehder and originated in Hungary and Iran. Other cluster Lindividuals had poor correlations and originated from Crimea, Macedonia, and Poland.

\section{Discussion}

Genetic analysis at 13 microsatellite loci revealed significant differentiation between the two wild subspecies considered the progenitors of the domesticated european pear. Morphological variation in fruit and floral characters did not distinguish these subspecies since the results were based on a limited number of characters. Moreover, the variation in trait values among wild populations for characters associated with domestication is often low and may be a poor predictor of genetic potential in these taxa (Tanksley and McCouch, 1997).

Pyrus communis ssp. caucasica individuals from the Caucasus, Ukraine, and Armenia group together (Fig. 2, clusters C-F) and may represent the wild center of diversity for domesticated pear, Pyrus communis ssp. communis. Pyrus communis ssp. pyraster individuals from Turkey, Macedonia and otherEuropean countries cluster separately in the network (Fig. 2, clusters G-L). These individuals display a high level of diversity that could have partially arisen through gene flow and introgressive hybridization with cooccurring congeneric species found in Europe. The differentiation of $P$. communis ssp. pyraster from $P$. communis ssp. caucasica could result from human migrations from the Trans-Caucasia region through Turkey and into the Balkan region over thousands of years (Gamkrelidze and Ivanov, 1990). In some cases, open pollinated sibling individuals did not differentiate into the same cluster. This is not surprising since seeds could have been fertilized by pollen from highly diverse trees in the wild.

Some individuals do not clearly fit into any of the 12 identified clusters. Hybridization is prevalent in Pyrus and some of these individuals appear to have a hybrid lineage, as indicated by the presence of serrated leaves. Many of the wild pears, particularly of European origin, could be escapes from cultivation or hybrids between wild $P$. communis and native european pear species, such as P. nivalis (Aldasoro et al., 1996; Paganova, 2003). More accurate determination of this would be possible with increased sampling in the region of the putative hybridization.

The broad diversity of the NPGS wild P. communis collection is evident through genetic analyses of molecular markers, but not readily apparent by morphological characterizations. Our study of 145 individual trees represents a conservative estimate of the natural diversity. Both the number and identity of alleles measured through allelic richness indicate that each genetic cluster, regardless of its size, adds measurable molecular diversity to the overall collection. Although leaves and fruits are morphologically similar, further morphological characterization of additional sibling populations will likely reveal novel phenotypic traits of interest to pear breeders. Increased sampling intensity, either by pursuing new collection trips or by sampling additional clones or seeds from previous collection trips from eastern Europe and Asia Minor, is critical to adequately evaluate natural genetic diversity of wild $P$. communis.

\section{Literature Cited}

Aldasoro, J.J., C. Aedo, and F. Munoz Garmendia. 1996. The genus Pyrus L. (Rosaceae) in south-west Europe and North Africa. Bot. J. Linnean Soc. 121:143-158. 
Bell, R.L. 1982. Pear genetics and germplasm-Priorities for breeding. Acta Hort. 124:13-20.

Bell, R.L. 1992. Additional east European Pyrus germplasm with resistance to pear Psylla nymphal feeding. HortScience 27:412-413.

Brooks, H.J. 1968. Collecting wild fruits in the USSR. HortScience 3:258-260.

Challice, J.S. and M.N. Westwood. 1973. Numerical taxonomic studies of the genus Pyrus using both chemical and botanical characters. Bot. J. Linnean Soc. 67:121-148.

El Mousadik,A. and R.H.Petit. 1996. High level of genetic differentiation for allelic richness among populations of the argan tree [Argania spinosa (L.) Skeels] endemic of Morocco. Theor. Appl. Genet. 92:832-839.

Gamkrelidze, T.V. and V.V. Ivanov. 1990. The early history of IndoEuropean languages. Sci. Amer. 262(3):110-116.

Goudet, J. 1995. FSTAT, a program for IBM PC compatibles to calculate Weir and Cockerham's (1984) estimators of F-statistics. J. Hered. 86:485-486.

Hayashi, T. and T. Yamamoto. 2002. Genome research on peach and pear. J. Plant Biotech. 4:45-52.

Hedrick, U.P. 1921. The pears of New York. J.B. Lyon, New York.

Hemmat, M., N.F. Weeden, and S.K. Brown. 2003. Mapping and evaluation of Malus $\times$ domestica microsatellites in apple and pear. J. Amer. Soc. Hort. Sci. 128:515-520.

Katayama, H. and C. Uematsu. 2003. Comparative analysis of chloroplast DNA in Pyrus species: Physical map and gene localization. Theor. Appl. Genet. 106:303-310.

Kimura, T., Y.Z. Shi, M. Shoda, K. Kotobuki, N. Matsuta, T. Hayashi, Y. Ban, and T. Yamamoto. 2002. Identification of Asian pear varieties by SSR analysis. Breeding Sci. 52:115-121.

Lewis, P.O. and D. Zaykin. 2001. GDA user's manual. 19 Oct. 2005. $<$ http://hydrodictyon.eeb.uconn.edu/people/plewis/software.php>.

Liebhard, R., L. Gianfranceschi, B. Koller, C.D. Ryder, R. Tarchini, E. Van de Weg, and C Gessler. 2002. Development and characterization of 140 new microsatellites in apple (Malus $\times$ domestica Borkh.). Mol. Breeding 10:217-241.

Liebhard, R., M. Kellerhals, W. Pfammatter, M. Jertmini, and C. Gessler. 2003. Mapping quantitative physiological traits in apple (Malus $\times$ domestica Borkh.). Plant Mol. Biol. 52:511-526.

Lombard, P., J. Hull, and M.N. Westwood. 1980. Pear cultivars of North America. Fruit Var. J. 34:74-83.

Nei, M. 1987. Molecular evolutionary genetics. Columbia University Press, New York.

Paganova, V. 2003. Taxonomic reliability of leaf and fruit morphological characteristics of the Pyrus L. taxa in Slovakia. Zahradnictvi (Horticultural Science, Prague) 30:98-107.

Pierantoni, L., K.-H. Cho, I.-S. Shin, R. Chiodini, S. Tartarini,L. Dondini, S.-J. Kang, and S. Sansavini. 2004. Characterisation and transferability of apple SSRs to two European pear $F_{1}$ populations. Theor. Appl. Genet. 109:1519-1524.

Pritchard, J.K., M.Stephens, and P. Donnelly. 2000. Inference of population structure using multilocus genotype data. Genetics 155:945-959.

Rehder, A. 1940. Manual of cultivated trees and shrubs hardy in North America, exclusive of the subtropical and warmer temperate regions. Macmillan, New York. Rosenberg, N.A., J.K. Pritchard, J.L. Weber, H.M. Cann, K.K. Kidd, L.A. Zhivotovsky, and M.W. Feldman. 2002. Genetic structure of human populations. Science 298:2381-2385.

Schneider, S., D. Roessli, and L. Excoffier. 2000. Arlequin ver. 2.000: A software for population genetics data analysis. Genetics and Biometry Laboratory, Univ. of Geneva, Switzerland.

Tanksley, S.D. and S.R. McCouch. 1997. Seed banks and molecular maps: Unlocking genetic potential from the wild. Science 277:1063-1066.

U.S. Department of Agriculture. 2005. Germplasm Resources Information Network-(GRIN). Accession area queries. USDA, ARS, National Genetic Resources Program. 19 Oct. 2005. <http://www.ars-grin.gov/ npgs/acc/acc_queries.html>.

van der Zwet, T., V. Cociu, B. Czarnecki, J. Nyeki, and J. Blazek. 1989. Collecting Pyrus germplasmin Romania, Poland, Hungary, and Czechoslovakia. HortScience 24:420-424.

van der Zwet, T., D. Stankovic, and V. Cociu. 1983. Collecting Pyrus germplasm in eastern Europe and its significance to the USDA pear breeding program. Acta Hort. 140:43-45.

Vavilov, N.I. 1994. Origin and geography of cultivated plants. D. Love (translator). Cambridge Univ. Press, Cambridge, England.

Westwood, M.N. and P.H. Westigard. 1969. Degree of resistance among pear species to the woolly pear aphid, Eriosoma pyricola. J. Amer. Soc. Hort. Sci. 94:91-93.

Yamamoto, T., T. Kimura, Y. Sawamura, K. Kotobuki, Y. Ban, T. Hayashi, and N. Matsuta. 2001. SSRs isolated from apple can identify polymorphism and genetic diversity in pear. Theor. Appl. Genet. 102:865-870.

Yamamoto, T., T. Kimura, M. Shoda, T. Imai, T. Saito, Y. Sawamura, K. Kotobuki, T. Hayashi, and N. Matsuta. 2002a. Genetic linkage maps constructed by using an interspecific cross between japanese and european pears. Theor. Appl. Genet. 106:9-18.

Yamamoto, T., T. Kimura, M. Shoda, Y. Ban, T. Hayashi, and N. Matsuta. 2002b. Development of microsatellite markers in the japanese pear (Pyrus pyrifolia Nakai). Mol. Ecol. Notes. 2:14-16.

Yamamoto, T., T. Kimura, Y. Sawamura, T. Manabe, K. Kotobuki, T. Hayashi, Y. Ban, and N. Matsuta. 2002c. Simple sequence repeats for genetic analysis in pear. Euphytica 124:129-137.

Yamamoto, T., T. Kimura, Y. Sawamura, C. Nishitani, S. Ohta, Y. Adachi, T. Hirabayashi, R. Liebhard, C. Gessler, W.E. van de Weg, and T. Hayashi. 2005. Genetic linkage maps of european and japanese pears. Plant Animal Genome XIII. p. 198. (Abstr.) 